\title{
A Luminescent Receptor with Affinity for $N$-terminal Histidine in Peptides in Aqueous Solution
}

Michael Kruppa, Christian Mandl, Stefan Miltschitzky and Burkhard König *

\section{Supporting Information}

1. Synthesis 2

2. Spectra and emission titrations 6

3. Quantum yields 11

\section{Synthesis}

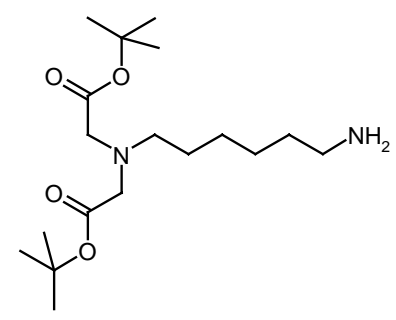

tert-Butyl-[(6-aminohexyl)-tert-butoxycarbonylmethylamino]-acetate (2): N-Z-1,6-diaminohexane $(\mathbf{1})^{1}(1.21 \mathrm{~g}, 4.9 \mathrm{mmol})$ was dissolved in $\mathrm{MeCN}(20 \mathrm{ml})$ and mixed with tert-butyl bromoacetate $(1.56 \mathrm{ml}, 10.6 \mathrm{mmol})$, potassium carbonate $(2.92 \mathrm{~g}, 21.1 \mathrm{mmol})$ and a spatula tip of potassium iodide. The suspension was stirred 2 days at $60{ }^{\circ} \mathrm{C}$ and monitored by TLC (ethyl acetate). The mixture was filtrated, diluted with $20 \mathrm{ml}$ of water and extracted twice with $30 \mathrm{ml}$ of ethyl acetate. After drying over sodium sulfate the organic solvents were evaporated to yield the crude product. Purification using column chromatography (silica, ethyl acetate, $R_{\mathrm{f}}$ $=0.9)$ gave the Z-protected IDA-intermediate as a colourless oil.

To deprotect the Z-group the oil was dissolved in ethanol ( $25 \mathrm{ml})$ and mixed with a spatula tip of $10 \% \mathrm{Pd} / \mathrm{C}$. Hydrogenolytical cleavage was performed in an autoclave at $10 \mathrm{bar}_{2}$ pressure for $12 \mathrm{~h}$ at RT. After filtration the ethanol was evaporated to yield 2 (4.3 mmol, $1.46 \mathrm{~g}, 87 \%$ ) as colourless oil. ${ }^{1} \mathrm{H}-\mathrm{NMR}\left(300 \mathrm{MHz} ; \mathrm{CDCl}_{3}\right): \delta=1.24-1.29\left(\mathrm{~m}, 4 \mathrm{H}, \mathrm{CH}_{2}\right), 1.38-1.49(\mathrm{~m}$, $\left.20 \mathrm{H}, \mathrm{CH}_{3}-\mathrm{Boc}+\mathrm{CH}_{2}\right), 2.62-2.69\left(\mathrm{~m}, 4 \mathrm{H}, \mathrm{CH}_{2}\right), 3.16-3.18\left(\mathrm{~m}, 2 \mathrm{H}, \mathrm{CH}_{2}\right), 3.40(\mathrm{~s}, 4 \mathrm{H}, \mathrm{N}-$ $\left.\mathrm{CH}_{2}\right), 4.82 \mathrm{ppm}(\mathrm{bs}, 1 \mathrm{H}, \mathrm{NH}) .-{ }^{13} \mathrm{C}-\mathrm{NMR}\left(75 \mathrm{MHz} ; \mathrm{CDCl}_{3}\right): \delta=26.6\left(-, 1 \mathrm{C}, \mathrm{CH}_{2}\right), 26.8(-$,

\footnotetext{
${ }^{1}$ Atwell, G. J.; Denny, W. A. Synthesis 1984, 12, 1032-1033.
} 
$\left.1 \mathrm{C}, \mathrm{CH}_{2}\right), 27.8\left(-, 1 \mathrm{C}, \mathrm{CH}_{2}\right), 28.2\left(+, 6 \mathrm{C}, \mathrm{CH}_{3}\right), 40.9$ (-, $\left.1 \mathrm{C}, \mathrm{CH}_{2}\right), 54.0\left(-, 1 \mathrm{C}, \mathrm{CH}_{2}\right), 55.9$ $\left(-, 2 \mathrm{C}, \mathrm{CH}_{2}\right), 66.6\left(-, 1 \mathrm{C}, \mathrm{CH}_{2}\right), 80.9\left(\mathrm{C}_{\text {quat }}, 2 \mathrm{C}, \mathrm{C}-\mathrm{Boc}\right), 170.8 \mathrm{ppm}\left(\mathrm{C}_{\text {quat }}, 2 \mathrm{C}, \mathrm{COO}^{t} \mathrm{Bu}\right) .-$ IR: $\tilde{v}=2930,1740,1714,1209 \mathrm{~cm}^{-1} .-\mathrm{MS}\left(\mathrm{CI}-\mathrm{MS}, \mathrm{NH}_{3}\right): \mathrm{m} / z(\%)=345(100)\left[\mathrm{MH}^{+}\right] .-$ HRMS $\left(\mathrm{C}_{18} \mathrm{H}_{36} \mathrm{~N}_{2} \mathrm{O}_{4}{ }^{+\bullet}\right)$ : calc. 344.2675 , found $344.2671 \pm 0.72$ ppm. - Elemental analysis calc. for $\mathrm{C}_{18} \mathrm{H}_{36} \mathrm{~N}_{2} \mathrm{O}_{4}$ x ethyl acetate: C 58.36, H 9.97, N 6.93; found C 58.29, H 9.92, N 6.98.

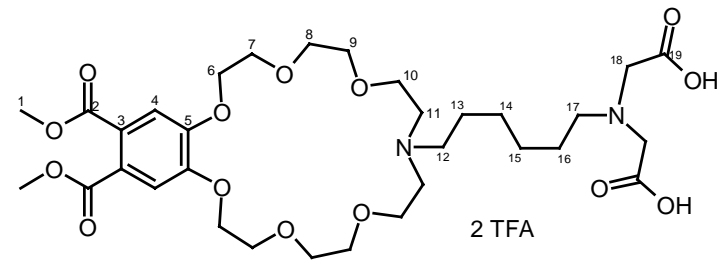

14-[6-(Bis-carboxymethyl-amino)-hexyl]-6,7,9,10,13,14,15,16,18,19,21,22-dodecahydro12H-5,8,11,17,20,23-hexaoxa-14-aza-benzocyclohenicosene-2,3-dicarboxylic acetic acid dimethylester ditriflate (4): Compound 2 (0.3 g, 0.9 mmol) was dissolved in $\mathrm{MeCN}$ (14 ml) and mixed with 4,5-bis-(2-\{2-[2-(toluol-4-sulfonyloxy)-ethoxy]-ethoxy\}-ethoxy)-phthalic acid dimethylester $(3)^{2}(0.67 \mathrm{~g}, 0.8 \mathrm{mmol})$, potassium carbonate $(1.29 \mathrm{~g}, 9.3 \mathrm{mmol})$ and potassium iodide $(0.14 \mathrm{~g}, 0.9 \mathrm{mmol})$. The suspension was refluxed for $2 \mathrm{~d}$ and the reaction progress monitored by $\mathrm{TLC}\left(\mathrm{CHCl}_{3}: \mathrm{MeOH}=6: 1\right)$. After cooling to room temp. the mixture was filtered over celite, washed with $\mathrm{MeCN}$ and $\mathrm{CHCl}_{3}$ and the filtrate was evaporated to dryness. Purification using column chromatography (silica, $\mathrm{CHCl}_{3}: \mathrm{MeOH}=20: 1 \rightarrow 15: 1, R_{\mathrm{f}}$ $\left.\left(\mathrm{CHCl}_{3}: \mathrm{MeOH}=6: 1\right)=0.33\right)$ gave bis tert-butyl protected 4 as a light yellow oil.

This diester was dissolved in TFA $(5 \mathrm{ml})$ and stirred for $2 \mathrm{~h}$ at room temp. After evaporating the TFA, the slurry was suspended in diethylether and decanted. Compound 4 ( $0.5 \mathrm{mmol}, 0.44$ g, 65\%) is a colourless hygroscopic solid. M.p.: $38-40{ }^{\circ} \mathrm{C} .-{ }^{1} \mathrm{H}-\mathrm{NMR}\left(600 \mathrm{MHz} ; \mathrm{D}_{2} \mathrm{O}\right.$; COSY, HSQC): $\delta=0.93-0.98$ (m, 2 H, H(15)), 1.01-1.04 (m, 2 H, H(14)), 1.41-1.46 (m, 4 H, $\mathrm{H}(13), \mathrm{H}(16)), 2.99-3.02$ (m, $2 \mathrm{H}, \mathrm{H}(12)), 3.07-3.10$ (m, $2 \mathrm{H}, \mathrm{H}(17)), 3.23-3.27$ (m, $2 \mathrm{H}$, $\mathrm{H}(11))$, 3.29-3.33 (m, 2 H, H(11)), 3.58-3.63 (m, $6 \mathrm{H}, \mathrm{H}(10), \mathrm{H}(8 / 9)), 3.66-3.74$ (m, $6 \mathrm{H}$, $\mathrm{H}(8 / 9)), 3.78$ (s, $6 \mathrm{H}, \mathrm{H}(1)), 3.77-3.80$ (m, 2 H, H(7)), 3.86-3.89 (m, 2 H, H(7)), 4.05 (s, 4 H, $\mathrm{H}(18)$ ), 4.05-4.08 (m, $2 \mathrm{H}, \mathrm{H}(6))$, 4.17-4.20 (m, $2 \mathrm{H}, \mathrm{H}(6))$, 7.18 ppm (s, $2 \mathrm{H}, \mathrm{H}(4))$ ) - ${ }^{13} \mathrm{C}-$ NMR (100 MHz; D 20 ; HSQC, HMBC): $\delta=22.6 \& 23.0$ (-, 2 C, C(14), C(15)), $25.0 \& 25.1$ (-, 2 C, C(13), C(16)), 53.2 (-, 1 C, C(12)), 53.2 (+, 2 C, C(1)), 53.7 (-, 2 C, C(11)), 54.4 (-, 2 C, C(18)), 56.4 (-, 1 C, C(17)), 63.7 (-, 2 C, C(10)), 68.2 (-, 2 C, C(6)), 68.9 (-, 2 C, C(7)), 69.8 (-, 4 C, C(8), C(9)), $112.6\left(+, 2\right.$ C, C(4)), $124.7\left(\mathrm{C}_{\text {quat }}, 2 \mathrm{C}, \mathrm{C}(3)\right), 149.6\left(\mathrm{C}_{\text {quat }}, 2 \mathrm{C}\right.$,

\footnotetext{
${ }^{2}$ C. Mandl, PhD thesis, Universität Regensburg, 2004.
} 
$\mathrm{C}(5)), 168.1\left(\mathrm{C}_{\text {quat }}, 2 \mathrm{C}, \mathrm{C}(2)\right), 169.3 \mathrm{ppm}\left(\mathrm{C}_{\text {quat }}, 2 \mathrm{C}, \mathrm{C}(19)\right)$ [TFA signals are not listed]. - IR (KBr): 3450, 2930, 2890, 1732, 1294, $1196 \mathrm{~cm}^{-1}$. - MS ((-)ESI, DCM/MeOH + $10 \mathrm{mmol} / 1$ $\left.\mathrm{NH}_{4} \mathrm{Ac}\right): m / z(\%)=685(100)\left[\mathrm{M}-\mathrm{H}^{+}\right] .-\mathrm{HRMS}\left(\mathrm{C}_{32} \mathrm{H}_{51} \mathrm{~N}_{2} \mathrm{O}_{14}{ }^{+}\right)$: calc. 687.3339, found $687.3340 \pm 0.64 \mathrm{ppm}$.

\section{Dipeptide Synthesis}

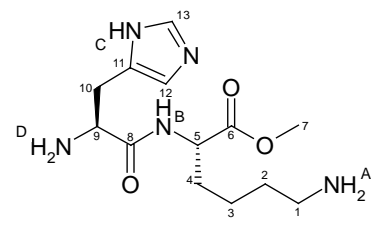

H-His-Lys-OMe 3 TFA

H-Lys(Boc)-OMe $\cdot \mathrm{HCl}(0.25 \mathrm{~g}, 0.8 \mathrm{mmol})$ was dissolved in DCM (15 ml) and DIPEA (0.43 $\mathrm{ml}, 2.5 \mathrm{mmol})$ under nitrogen atmosphere. Boc-His(Boc)-OH·dicyclohexylamine $(0.49 \mathrm{~g}$ $0.9 \mathrm{mmol})$, EDC $(0.18 \mathrm{ml}, 1.0 \mathrm{~mol})$ and $\mathrm{HOBt}(0.14 \mathrm{~g}, 1.0 \mathrm{mmol})$ were added and the reaction was monitored by TLC (ethyl acetate). After $12 \mathrm{~h}$ the solution was mixed with water $(15 \mathrm{ml})$, diluted with DCM $(20 \mathrm{ml})$ and extracted twice with brine $(20 \mathrm{ml})$. The combined organic layers were dried over sodium sulfate and the solvent evaporated. The crude product was purified using column chromatography (silica, ethyl acetate, $R_{\mathrm{f}}=0.4$ ) resulting in a colourless solid.

The protected dipeptide was dissolved in a small amount of DCM and mixed with TFA $(1.46 \mathrm{ml}, 18.9 \mathrm{mmol})$. After $12 \mathrm{~h}$ the mixture was evaporated to dryness and completion of the reaction controlled by NMR. The triflate salt was redissolved in water, washed with DCM and lyophilised. H-His-Lys-OMe 3 TFA $(0.29 \mathrm{~g}, 0.5 \mathrm{mmol}, 58 \%)$ is a colourless, hygroscopic solid. M.p.: $62-63^{\circ} \mathrm{C} .-{ }^{1} \mathrm{H}-\mathrm{NMR}\left(600 \mathrm{MHz}\right.$; DMSO-d $\left.{ }_{6}\right): \delta=1.16-1.18$ (m, $2 \mathrm{H}$, COSY: H(3)), 1.34-1.59 (m, 2 H, HSQC: H(2)), 1.60-1.76 (m, 2 H, COSY: H(4)), 2.76 (m, 2 H, COSY: H(1)), $3.19\left(2 \mathrm{x} \mathrm{dd},{ }^{2} J(\mathrm{H}, \mathrm{H})=15.4 \mathrm{~Hz},{ }^{3} J(\mathrm{H}, \mathrm{H})=7.2 \mathrm{~Hz}, 2 \mathrm{H}, \mathrm{COSY}: \mathrm{H}(10)\right), 3.62$ (s, 3 H, HSQC: H(7)), $4.22\left(\mathrm{t},{ }^{3} J(\mathrm{H}, \mathrm{H})=6.9 \mathrm{~Hz}, 1 \mathrm{H}, \mathrm{COSY}: \mathrm{H}(9)\right), 4.29\left(\mathrm{dt},{ }^{3} J(\mathrm{H}, \mathrm{H})=5.4\right.$, $7.8 \mathrm{~Hz}, 1 \mathrm{H}, \mathrm{COSY}: \mathrm{H}(5)), 7.41$ (s, $1 \mathrm{H}, \mathrm{HMBC}$ : H(12)), 7.90 (bs, 3 H, HMBC: $\mathrm{H}_{\mathrm{A}}$ ), 8.118.80 (bs, $\left.1 \mathrm{H}, \mathrm{HMBC}: \mathrm{H}_{\mathrm{D}}\right) 8.92\left(\mathrm{~d},{ }^{3} J(\mathrm{H}, \mathrm{H})=7.3 \mathrm{~Hz}, 1 \mathrm{H}, \mathrm{HMBC}: \mathrm{H}_{\mathrm{B}}\right), 8.98$ (s, $1 \mathrm{H}, \mathrm{HMBC}$ : $\mathrm{H}(13)$ ), 13.50-15.45 ppm (bs, $2 \mathrm{H}, \mathrm{H}_{\mathrm{C}}$ ). $-{ }^{13} \mathrm{C}-\mathrm{NMR}$ (150 MHz; DMSO-d ${ }_{6}$; HSQC, HMQC): $\delta=22.0(-, 1 \mathrm{C}, \mathrm{C}(3)), 26.4(-, 1 \mathrm{C}, \mathrm{C}(10)), 26.5$ (-, $1 \mathrm{C}, \mathrm{C}(2)), 30.4(-, 1 \mathrm{C}, \mathrm{C}(4)), 38.4(-, 1$ C, C(1)), $51.2(+, 1 \mathrm{C}, \mathrm{C}(9)), 52.0(+, 1 \mathrm{C}, \mathrm{C}(5)), 52.1(+, 1 \mathrm{C}, \mathrm{C}(7)), 117.8(+, 1 \mathrm{C}, \mathrm{C}(12))$, $127.2\left(\mathrm{C}_{\text {quat }}, 1 \mathrm{C}, \mathrm{C}(11)\right), 134.3$ (+, $\left.1 \mathrm{C}, \mathrm{C}(13)\right), 167.5\left(\mathrm{C}_{\text {quat }}, 1 \mathrm{C}, \mathrm{C}(8)\right), 171.7 \mathrm{ppm}\left(\mathrm{C}_{\text {quat }}, 1 \mathrm{C}\right.$, C(6)) [TFA signals are not listed]. - IR (KBr): $\tilde{v}=3431,3123,3049$ 2965, 1676, 1204, 1135, 
$723 \mathrm{~cm}^{-1}$. - MS (ESI, $\left.\mathrm{H}_{2} \mathrm{O} / \mathrm{MeOH}+10 \mathrm{mmol} / \mathrm{l} \mathrm{NH} \mathrm{N}_{4} \mathrm{Ac}\right): \mathrm{m} / z(\%)=298(100)\left[\mathrm{MH}^{+}\right], 170$ (10) $\left[\mathrm{M}+2 \mathrm{H}^{+}+\mathrm{MeCN}\right]$. - Elemental analysis calc. for $\mathrm{C}_{19} \mathrm{H}_{26} \mathrm{~N}_{5} \mathrm{O}_{9} \mathrm{~F}_{9} \times \mathrm{H}_{2} \mathrm{O}$ : C 34.71, H 4.29, N 10.65; found: C 34.17, H 4.28, N 10.55.

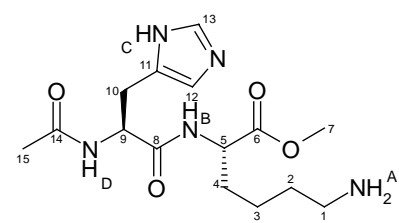

Ac-His-Lys-OMe $\cdot 2$ TFA

H-Lys(Boc)-OMe $\cdot \mathrm{HCl}(0.25 \mathrm{~g}, 0.8 \mathrm{mmol})$ was dissolved in DCM (15 ml) and DIPEA (0.43 $\mathrm{ml}, 2.5 \mathrm{mmol})$ under nitrogen atmosphere. Ac-His(1-Trt)-OH (0.4 g $0.9 \mathrm{mmol})$, EDC (0.18 $\mathrm{ml}, 1.0 \mathrm{~mol})$ and HOBt $(0.14 \mathrm{~g}, 1.0 \mathrm{mmol})$ were added thereafter and the reaction was monitored by TLC (ethyl acetate). After $12 \mathrm{~h}$ the solution was mixed with water $(15 \mathrm{ml})$, diluted with DCM $(20 \mathrm{ml})$ and extracted twice with brine $(20 \mathrm{ml})$. The combined organic layers were dried over sodium sulfate and the solution evapurated. The crude product was purified using column chromatography (silica, ethyl acetate $\rightarrow$ ethyl acetate $/ \mathrm{MeOH}=1 / 1, \mathrm{R}_{\mathrm{f}}$ $($ ethyl acetate $)=0.1)$ resulting in a colourless solid.

The protected dipeptide was dissolved in a small amount of DCM and mixed with TFA $(1.06 \mathrm{ml}, 13.7 \mathrm{mmol})$. After $12 \mathrm{~h}$ the mixture was evaporated to dryness and completion of the reaction was controlled by NMR. The triflate salt was redissolved in water, washed with DCM and lyophilised. Ac-His-Lys-OMe $\cdot 2$ TFA (0.42 g, $0.7 \mathrm{mmol}, 93 \%)$ is a colourless, hygroscopic solid.

${ }^{1} \mathrm{H}-\mathrm{NMR}\left(600 \mathrm{MHz}\right.$; DMSO-d ${ }_{6}$ ): $\delta=1.28-1.41$ (m, $2 \mathrm{H}$, COSY: H(3)), 1.45-1.58 (m, $2 \mathrm{H}$, HSQC: H(2)), 1.59-1.78 (m, 2 H, COSY: H(4)), 1.83 (s, 3 H, HSQC: H(15)), 2.70-2.82 (m, $2 \mathrm{H}, \mathrm{COSY}: \mathrm{H}(1)), 2.85-3.11\left(2 \mathrm{x} \mathrm{dd},{ }^{2} J(\mathrm{H}, \mathrm{H})=14.9 \mathrm{~Hz},{ }^{3} J(\mathrm{H}, \mathrm{H})=5.5 \mathrm{~Hz}, 2 \mathrm{H}, \mathrm{COSY}\right.$ : H(10)), 3.62 (s, 3 H, HSQC: H(7)), 4.17-4.28 (m, 1 H, COSY: H(9)), 4.57-4.67 (m, $1 \mathrm{H}$, COSY: H(5)), 7.31 (s, 1 H, HMBC: H(12)), 7.81 (bs, 3 H, HMBC: $\left.\mathrm{H}_{\mathrm{A}}\right), 8.23\left(\mathrm{~d},{ }^{3} J(\mathrm{H}, \mathrm{H})=\right.$ $\left.7.9 \mathrm{~Hz}, 1 \mathrm{H}, \mathrm{HMBC}: \mathrm{H}_{\mathrm{D}}\right) 8.41\left(\mathrm{~d},{ }^{3} J(\mathrm{H}, \mathrm{H})=7.5 \mathrm{~Hz}, 1 \mathrm{H}, \mathrm{HMBC}: \mathrm{H}_{\mathrm{B}}\right), 8.94-8.98(\mathrm{~m}, 1 \mathrm{H}$, HMBC: H(13)), 14.01-14.73 ppm (bs, $2 \mathrm{H}, \mathrm{H}_{\mathrm{C}}$ ). $-{ }^{13} \mathrm{C}-\mathrm{NMR}(150 \mathrm{MHz}$; DMSO-d 6 ; HSQC, HMQC): $\delta=22.1(-, 1 \mathrm{C}, \mathrm{C}(3)), 22.4$ (+, $1 \mathrm{C}, \mathrm{C}(15)), 26.3(-, 1 \mathrm{C}, \mathrm{C}(2)), 27.0(-, 1 \mathrm{C}$, C(10)), 30.0 (-, 1 C, $\mathrm{C}(4)), 38.4$ (-, $1 \mathrm{C}, \mathrm{C}(1)), 51.3$ (+, $1 \mathrm{C}, \mathrm{C}(5)), 51.7$ (+, $1 \mathrm{C}, \mathrm{C}(9)), 51.8$ (+, 1 C, $\mathrm{C}(7)), 116.6$ (+, $1 \mathrm{C}, \mathrm{C}(12)), 129.3$ (C $\left.\mathrm{C}_{\text {quat }}, 1 \mathrm{C}, \mathrm{C}(11)\right), 133.6(+, 1 \mathrm{C}, \mathrm{C}(13)), 169.4$ $\left(\mathrm{C}_{\text {quat }}, 1 \mathrm{C}, \mathrm{C}(14)\right), 170.3\left(\mathrm{C}_{\text {quat }}, 1 \mathrm{C}, \mathrm{C}(8)\right), 172.1\left(\mathrm{C}_{\text {quat }}, 1 \mathrm{C}, \mathrm{C}(6)\right) .-\mathrm{IR}(\mathrm{KBr}): \tilde{v}=3431$, 
3122, 3050 2965, 2913, 1676, 1204, $1130 \mathrm{~cm}^{-1}$. - MS (ESI, $\left.\mathrm{H}_{2} \mathrm{O} / \mathrm{MeCN}\right): \mathrm{m} / \mathrm{z}(\%)=340$ (100) $\left[\mathrm{MH}^{+}\right]$.

\section{Metal Complexation}

To a solution of the diacid 4 (90 mg, $0.1 \mathrm{mmol})$ in water $(5 \mathrm{ml})$ was added $\mathrm{CuCl}_{2}(13 \mathrm{mg}$, $0.1 \mathrm{mmol})$ in $\mathrm{MeOH}(10 \mathrm{ml})$. Stirring was continued at room temp. for $2 \mathrm{~h}$. The solvent was evaporated and the residue dissolved in a small amount of water. Methanol was added until a precipitate was formed and the solid was removed by filtration. Methanol was evaporated and the crude product was recrystallised from methanol yielding compound 5 (73 $\mathrm{mg}, 0.09 \mathrm{mmol}$, $86 \%)$ as a blue solid.

m.p. $>200{ }^{\circ} \mathrm{C} .-\mathrm{MS}\left(\mathrm{ESI}, \mathrm{MeOH}+10 \mathrm{mmol} / 1 \mathrm{NH}_{4} \mathrm{Ac}\right): \mathrm{m} / z(\%)=847$ (34) $\left[\mathrm{MH}^{+}+2 \mathrm{MeCN}\right.$ $\left.+\mathrm{H}_{2} \mathrm{O}\right], 830(50)\left[\mathrm{MH}^{+}+2 \mathrm{MeCN}\right], 825(65)\left[\mathrm{MH}^{+}+\mathrm{H}_{2} \mathrm{O}+\mathrm{HAc}\right], 808$ (35) $\left[\mathrm{MH}^{+}+\mathrm{HAc}\right]$, $748(100)\left[\mathrm{MH}^{+}\right]$.

\section{Glossary}

Z: $\quad$ Z-(Zervas) amino protecting group

DCM: dichloromethane

TFA: trifluoro acetic acid

DIPEA: diisopropyl ethyl amine

EDC: 1-Ethyl-3-(3-dimethyllaminopropyl)carbodiimide hydrochloride

IDA: Iminodiacetic acid

HOBt: Hydroxybenzotriazole 
II. Spectra and emission titration in methanol

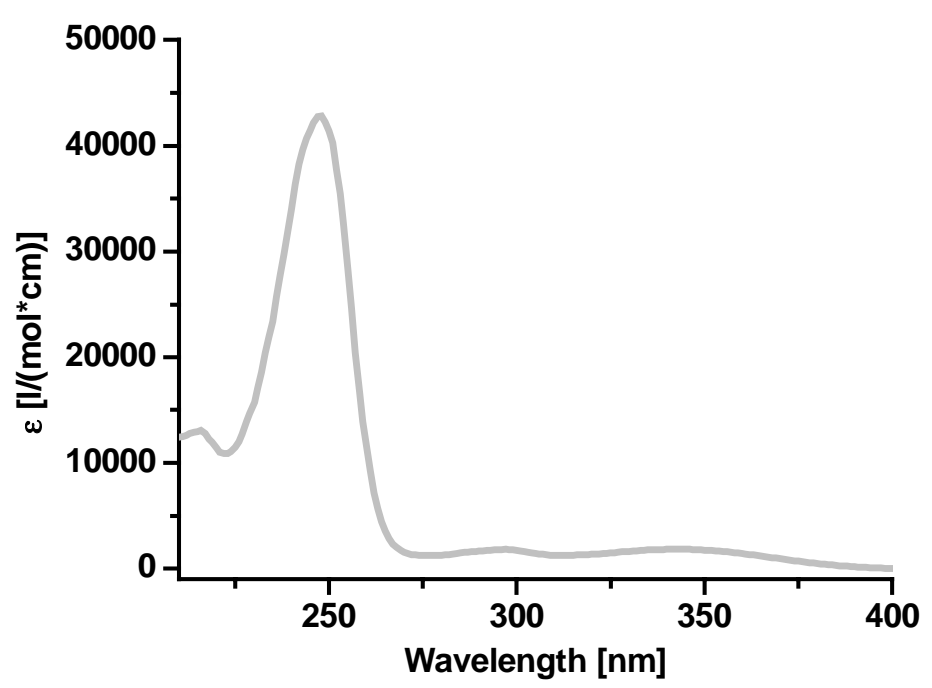

Figure S-1. Absorption spectra of compound 5 in methanol.

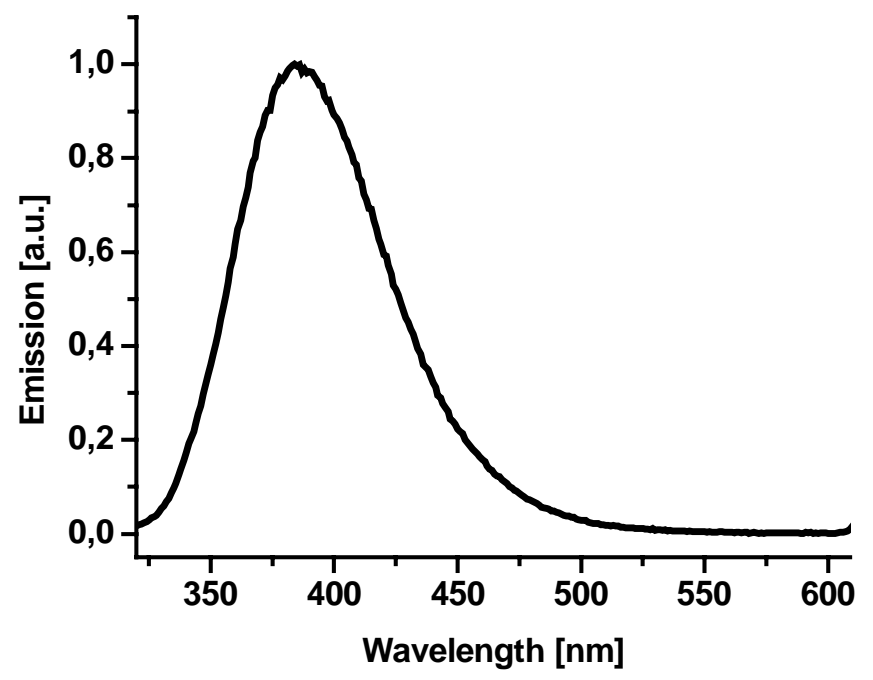

Figure S-2. Emission spectra of compound 5.in methanol (excitation at $270 \mathrm{~nm}$ ). 


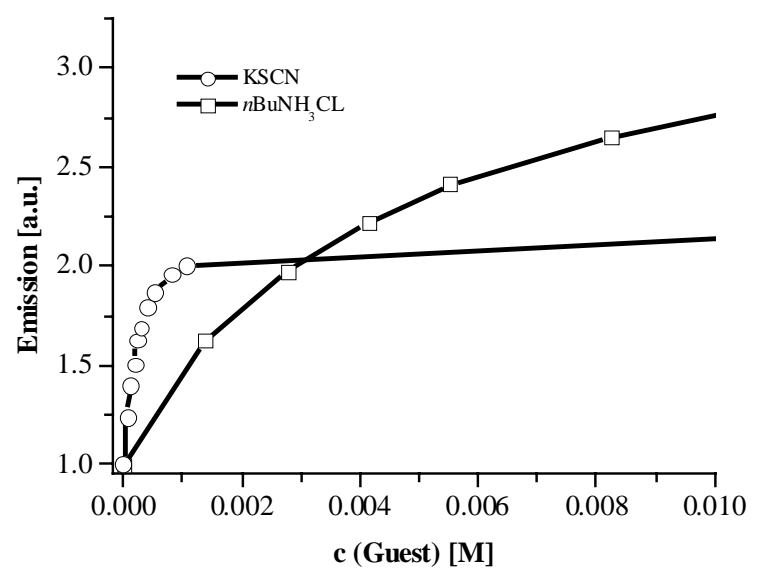

Figure S-3. Change in emission intensity of compound 6 at $384 \mathrm{~nm}$ upon titration with KSCN or $\mathrm{nBuNH}_{3} \mathrm{Cl}$ in methanol.

\section{Emission titrations in aqueous buffer}

Cary Eclipse Spectometer

Spectrometer setup:

Excitation wavelength $\lambda=305 \mathrm{~nm}$

Detection wavelength $\lambda=377-380 \mathrm{~nm}$

Temperature: $\mathrm{T}=298 \mathrm{~K}$

Titration conditions:

Solvent: $50 \mathrm{mM}$ HEPES buffer, $\mathrm{pH} 7.5$,

Starting volume: $2.5 \mathrm{ml}$

$\mathrm{c}$ (receptor) $=5.04 \cdot 10^{-5} \mathrm{M}$

$\mathrm{c}($ analyte $)=1.1-1.2 \cdot 10^{-2} \mathrm{M}$

Procedure:

The cuvette with $2.5 \mathrm{ml}$ of receptor 5 in HEPES buffer was titrated stepwise with small amounts $(10-50 \mu \mathrm{L}$; depending on the initial emission response) of the analyte solution. 


\section{Compound 5 vs. H-His-Lys-OMe}

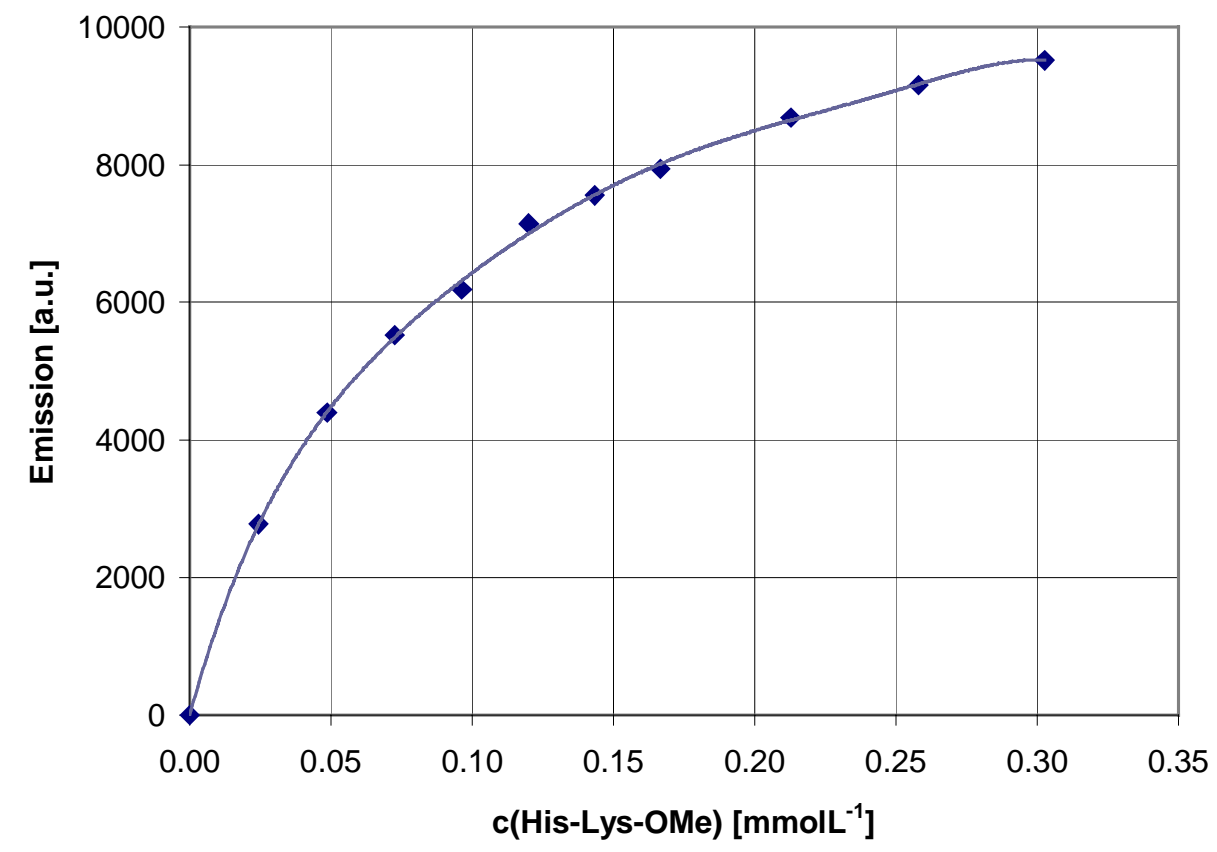

$\log K=4.2 \pm 0.1$

Job's-plot analysis:

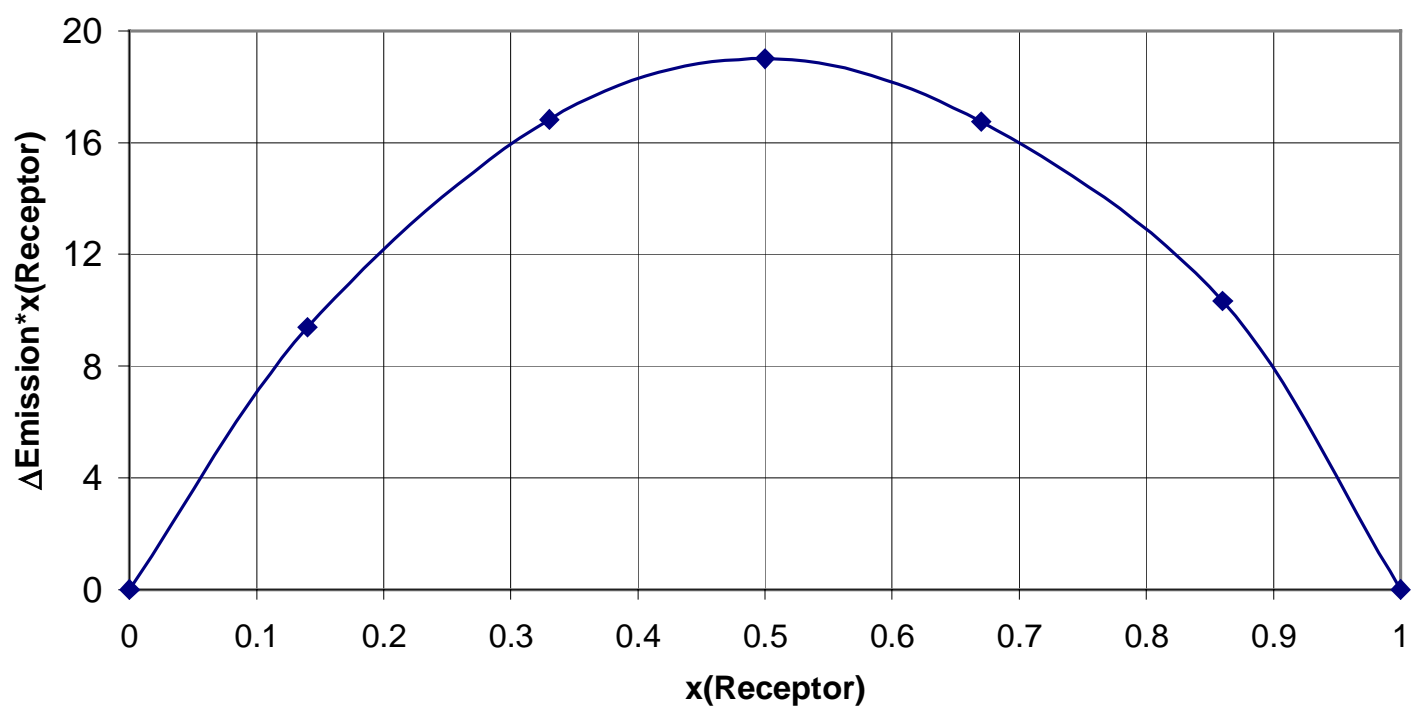




\section{Compound 5 vs. H-His-OMe}

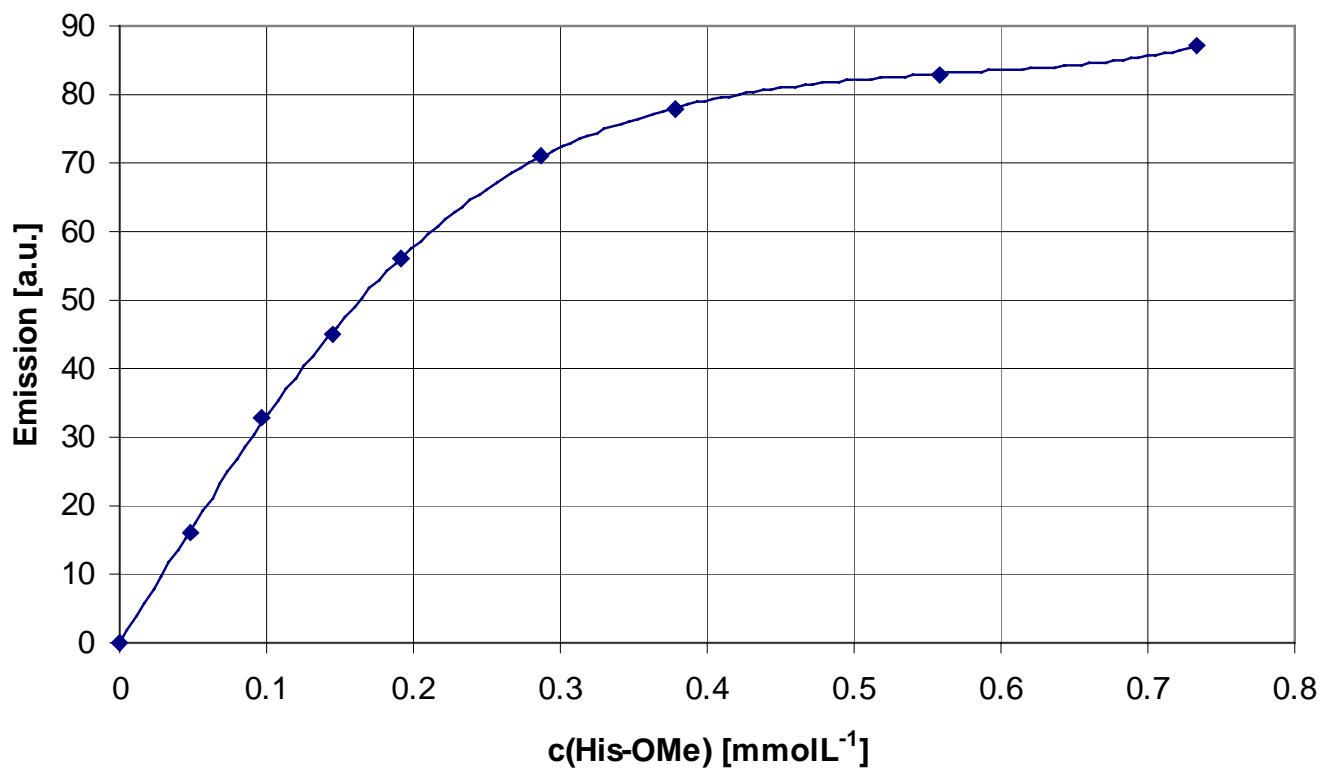

$\log K=3.8 \pm 0.1$

Job's-plot analysis:

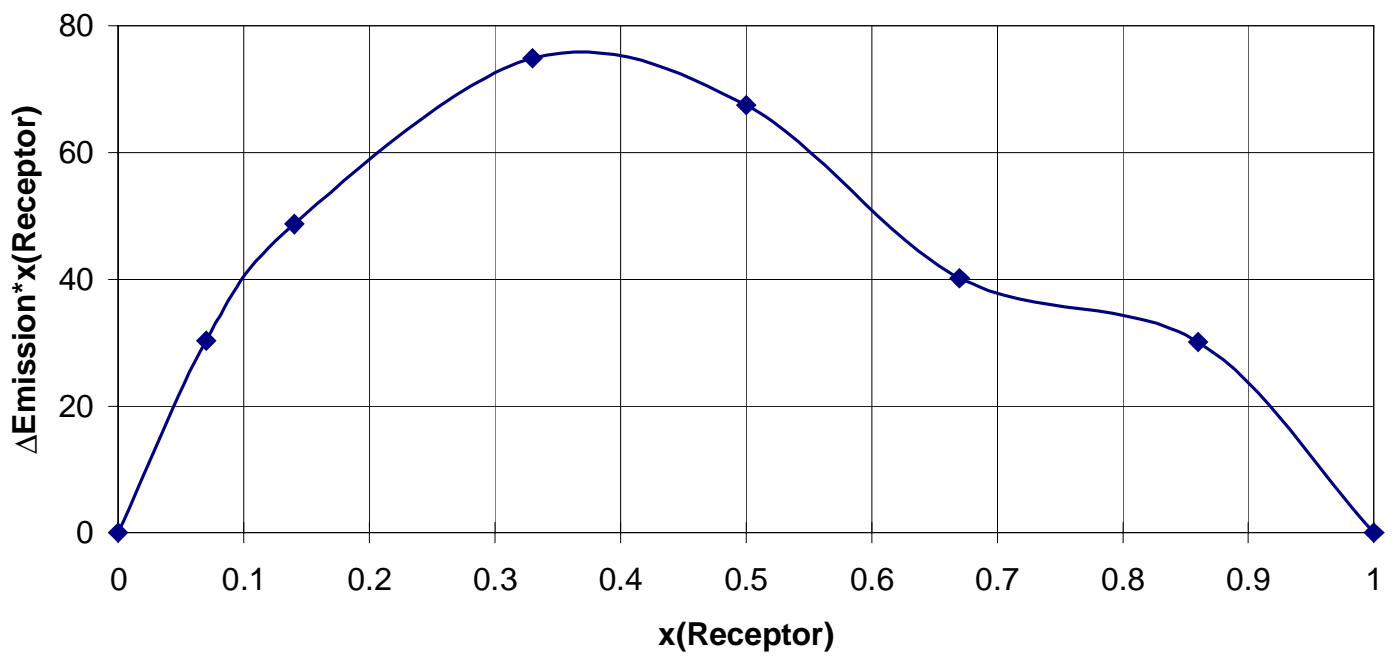




\section{Compound 5 vs. H-His-Gly-Gly-OH}

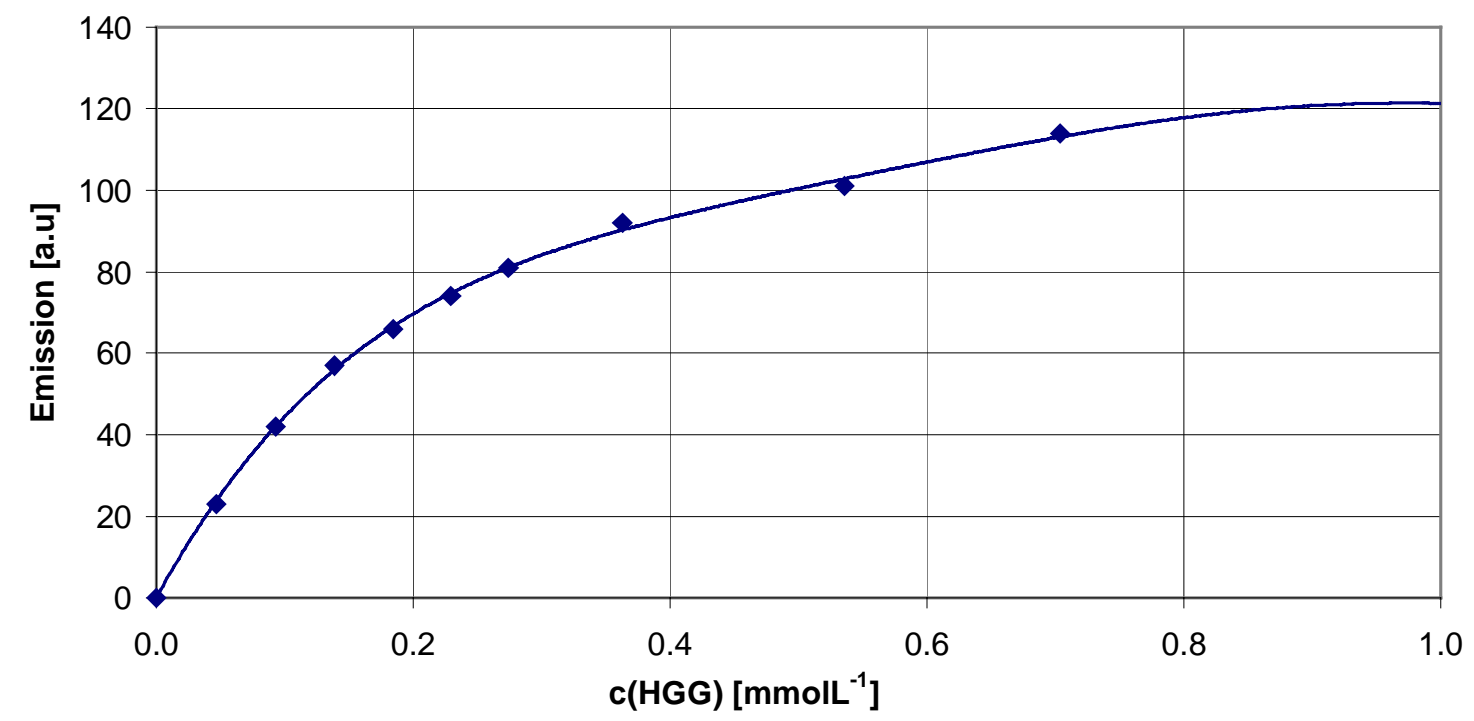

$\log \mathrm{K}=3.7 \pm 0.05$

Job's-plot analysis:

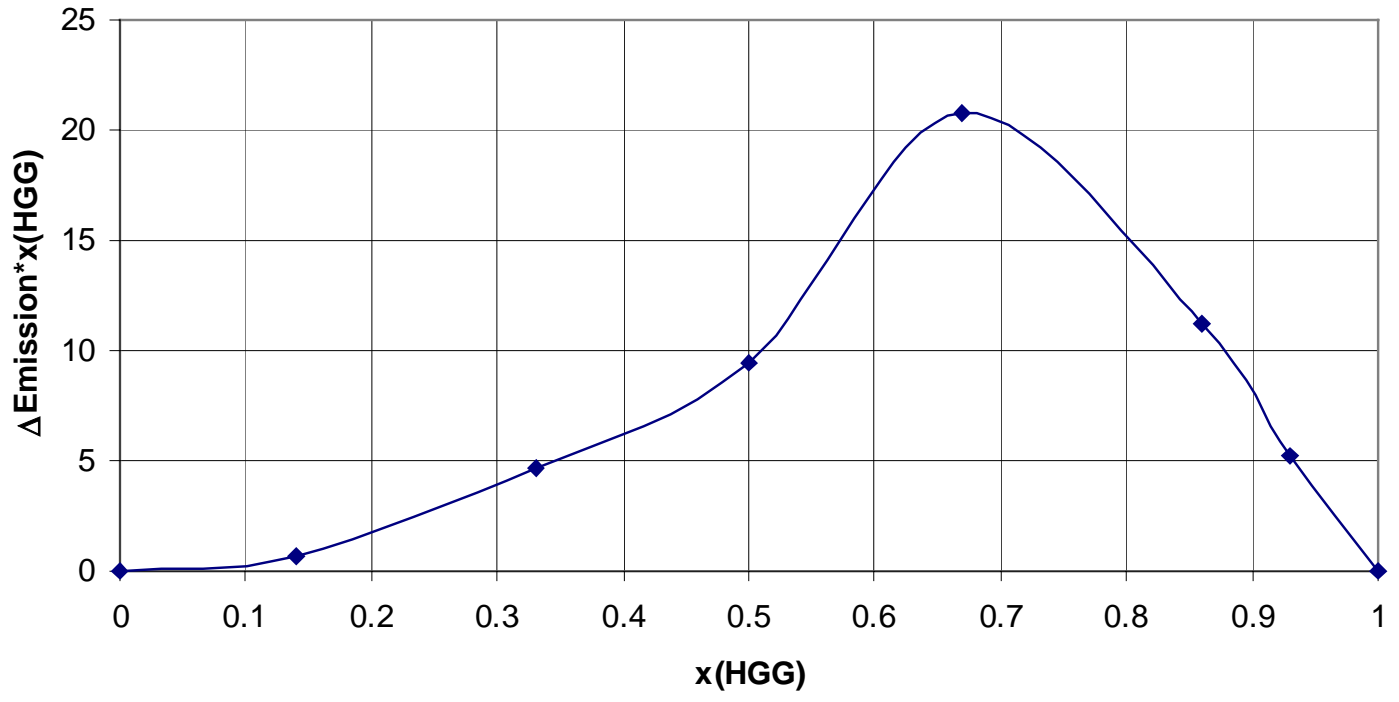




\section{Quantum yields}

All quantum yields were determined with quinine disulfate in $1 \mathrm{~N} \mathrm{H}_{2} \mathrm{SO}_{4}$ as the reference.

Compound 4

Compound 5

Compound $\mathbf{5}+$ His

Compound 5 + His-Lys-OMe

Compound 6

$$
\begin{aligned}
& \Phi=0.62 \\
& \Phi=0.0045 \\
& \Phi=0.012 \\
& \Phi=0.013 \\
& \Phi=0.09
\end{aligned}
$$

